\title{
Approximation by Discrete Singular Operators
}

\author{
George A. Anastassiou \\ University of Memphis \\ Department of Mathematical Sciences \\ Memphis, TN 38152, U.S.A. \\ ganastss@memphis. edu
}

\begin{abstract}
Here we study basic approximation properties with rates of our discrete versions of Picard, Gauss-Weierstrass, Poisson-Cauchy singular operators and of two other discrete operators. We prove uniform convergence of these operators to the unit operator. Also all these operators fulfill the global smoothness preservation property. The discussed operators act on the space of uniformly continuous functions over the real line.
\end{abstract}

\section{RESUMEN}

Aquí estudiamos las propiedades de aproximación básica con cocientes de nuestras versiones discretas de operadores singulares de Picard, Gauss-Weierstrass, PoissonCauchy y de otros dos operadores discretos. Probamos la convergencia uniforme de estos operadores al operador unitario. Además, todos estos operadores satisfacen la propiedad de preservación de suavidad global. Los operadores discutidos actúan sobre el espacio de funciones uniformemente continua sobre la recta real.

Keywords and Phrases: Discrete singular operator, modulus of continuity, uniform convergence, global smoothness.

2010 AMS Mathematics Subject Classification: 26A15, 26D15, 41A17, 41A25, 41A44. 


\section{Preliminaries}

Let $f: \mathbb{R} \rightarrow \mathbb{R}$ be a function which is uniformly continuous $\left(f \in C_{u}(\mathbb{R})\right.$ ). Following $[2$, p. 40-41, we define the first modulus of continuity,

$$
\omega_{1}(f, t):=\sup _{\substack{x, y \in \mathbb{R} \\|x-y| \leq t}}|f(x)-f(y)|, \quad t \geq 0 .
$$

The function $\omega_{1}$ is continuous at $t=0$ if and only if $f$ is uniformly continuous on $\mathbb{R}$. So that here $\omega_{1}(f, t) \rightarrow \omega_{1}(f, 0)=0$, as $t \rightarrow 0$. It also holds

$$
\omega_{1}(f, \lambda t) \leq(\lambda+1) \omega_{1}(f, t), \quad \lambda \geq 0
$$

Clearly $\omega_{1}(f, t)$ is finite for each $t \geq 0$.

In [1] we studied extensively the convergence to the unit operator of various integral singular operators. Here we define the discrete analogs of these operators next, and we study their uniform convergence to the unit operator with rates.

Let $0<\xi \leq 1$, such that $\xi \rightarrow 0+, x \in \mathbb{R} ; \frac{1}{\xi} \geq 1$.

i) We define the discrete Picard operators:

$$
\left(P_{\xi}^{*} f\right)(x):=\frac{\sum_{v=-\infty}^{\infty} f(x+v) e^{-\frac{|v|}{\xi}}}{\sum_{v=-\infty}^{\infty} e^{-\frac{|v|}{\xi}}} .
$$

ii) We define the discrete Gauss-Weierstrass operators:

$$
\left(W_{\xi}^{*} f\right)(x):=\frac{\sum_{v=-\infty}^{\infty} f(x+v) e^{-\frac{v^{2}}{\xi}}}{\sum_{v=-\infty}^{\infty} e^{-\frac{v^{2}}{\xi}}} .
$$

iii) We define the general discrete Poisson-Cauchy operators:

let $\alpha \in \mathbb{N}, \beta>\frac{1}{\alpha}$;

$$
\left(M_{\xi}^{*} f\right)(x):=\frac{\sum_{v=-\infty}^{\infty} f(x+v)\left(v^{2 \alpha}+\xi^{2 \alpha}\right)^{-\beta}}{\sum_{v=-\infty}^{\infty}\left(v^{2 \alpha}+\xi^{2 \alpha}\right)^{-\beta}} .
$$

iv) We define the basic discrete convolution operators:

let $\varphi: \mathbb{R} \rightarrow \mathbb{R}$, with $\|\varphi\|_{\infty}:=\sup _{x \in \mathbb{R}}|\varphi(x)| \leq K, K>0, \beta \in \mathbb{N}-\{1\}$;

$$
\left(\theta_{\xi}^{*} f\right)(x):=\frac{f(x)+\sum_{v \in \mathbb{Z}-\{0\}} f(x+v)\left(\frac{\varphi\left(\frac{v}{\xi}\right)}{\frac{v}{\xi}}\right)^{2 \beta}}{1+\sum_{v \in \mathbb{Z}-\{0\}}\left(\frac{\varphi\left(\frac{v}{\xi}\right)}{\frac{v}{\xi}}\right)^{2 \beta}} .
$$


v) We define the general discrete convolution operators:

let $\varphi: \mathbb{R} \rightarrow \mathbb{R}_{+}$with $\varphi(x) \leq A x^{2 \beta}, \forall x \in \mathbb{R}, \beta \in \mathbb{N}-\{1\}, A>0$;

$$
\left(T_{\xi}^{*} f\right)(x):=\frac{f(x)+\sum_{v \in \mathbb{Z}-\{0\}} f(x+v) \frac{\varphi\left(\frac{v}{\xi}\right)}{\left(\frac{v}{\xi}\right)^{4 \beta}}}{1+\sum_{v \in \mathbb{Z}-\{0\}} \frac{\varphi\left(\frac{v}{\varepsilon}\right)}{\left(\frac{v}{\xi}\right)^{4 \beta}}} .
$$

The above operators, as we will see, are well defined and are linear, positive, and bounded when $\|f\|_{\infty}:=\sup _{x \in \mathbb{R}}|f(x)|<\infty$. Furthermore

$$
P_{\xi}^{*}(1)=W_{\xi}^{*}(1)=M_{\xi}^{*}(1)=\theta_{\xi}^{*}(1)=T_{\xi}^{*}(1)=1,
$$

with

$$
\left\|P_{\xi}^{*}\right\|=\left\|W_{\xi}^{*}\right\|=\left\|M_{\xi}^{*}\right\|=\left\|\theta_{\xi}^{*}\right\|=\left\|T_{\xi}^{*}\right\|=1,
$$

on continuous bounded functions.

In this article we are motivated by $[3]$.

\section{Main Results}

All here as in Preliminaries earlier. We start with the basic approximation properties of discrete Picard operators.

We present

Theorem 2.1. It holds

$$
\left\|P_{\xi}^{*} f-f\right\|_{\infty} \leq\left[\frac{1+2 e^{-\frac{1}{\xi}}\left(2 \xi+2+\frac{1}{\xi}\right)}{1+2 \xi e^{-\frac{1}{\xi}}}\right] \omega_{1}(f, \xi) .
$$

The constant in the right hand side of (10) converges to 1 as $\xi \rightarrow 0+$. So that $\mathrm{P}_{\xi}^{*} \rightarrow$ I (unit operator), uniformly with rates, as $\xi \rightarrow 0+$.

Proof. We will use a lot

$$
\sum_{v=1}^{\infty} \frac{1}{v^{2}}=\frac{\pi^{2}}{6} \quad \text { (Euler, 1741). }
$$

We see that

$$
\sum_{v=-1}^{-\infty} e^{-\frac{|v|}{\xi}}=\sum_{v=1}^{\infty} e^{-\frac{v}{\xi}}<\sum_{v=1}^{\infty} \frac{1}{v^{2}}=\frac{\pi^{2}}{6}
$$

it converges. 
Thus

$$
\sum_{v=-\infty}^{\infty} e^{-\frac{|v|}{\xi}}=2 \sum_{\nu=1}^{\infty} e^{-\frac{v}{\xi}}+1<\frac{\pi^{2}}{3}+1
$$

Using 4] we obtain

$$
\sum_{\nu=1}^{\infty} e^{-\frac{v}{\varepsilon}}-e^{-\frac{1}{\varepsilon}} \leq \int_{1}^{\infty} e^{-\frac{v}{\varepsilon}} d v \leq \sum_{\nu=1}^{\infty} e^{-\frac{v}{\varepsilon}}
$$

Hence

$$
2 \int_{1}^{\infty} e^{-\frac{v}{\xi}} d v+1 \leq 2 \sum_{\nu=1}^{\infty} e^{-\frac{v}{\xi}}+1=\sum_{\nu=-\infty}^{\infty} e^{-\frac{|\nu|}{\xi}}
$$

Thus

$$
0<\frac{1}{\sum_{\nu=-\infty}^{\infty} e^{-\frac{|v|}{\xi}}} \leq \frac{1}{2 \int_{1}^{\infty} e^{-\frac{v}{\xi}} \mathrm{d} v+1}=\frac{1}{2 \xi e^{-\frac{1}{\xi}}+1} \rightarrow 1 \text {, as } \xi \rightarrow 0+.
$$

We need to prove that $g(v)=v e^{-\frac{v}{\varepsilon}}$ is decreasing for $v \geq 1$. Indeed we have that $g^{\prime}(v)=$ $e^{-\frac{v}{\xi}}\left(1-\frac{v}{\xi}\right) \leq 0$, by $\xi \leq 1 \leq v$.

So that, again by [4], we get that

$$
1+2 \sum_{v=1}^{\infty}\left(1+\frac{v}{\xi}\right) e^{-\frac{v}{\xi}} \leq 1+2\left[\int_{1}^{\infty}\left(1+\frac{v}{\xi}\right) e^{-\frac{v}{\xi}} d v+\left(1+\frac{1}{\xi}\right) e^{-\frac{1}{\xi}}\right]=:(*)
$$

Using integration by parts we have

$$
\int_{\frac{1}{\xi}}^{\infty} x e^{-x} d x=-\left.e^{-x}(x+1)\right|_{\frac{1}{\xi}} ^{\infty}=e^{-\frac{1}{\xi}}\left(\frac{1}{\xi}+1\right) .
$$

Hence we get

$$
\begin{gathered}
\int_{1}^{\infty}\left(1+\frac{v}{\xi}\right) e^{-\frac{v}{\xi}} d v=\int_{1}^{\infty} e^{-\frac{v}{\xi}} d v+\int_{1}^{\infty} \frac{v}{\xi} e^{-\frac{v}{\xi}} d v= \\
\xi e^{-\frac{1}{\xi}}+\xi \int_{\frac{1}{\xi}}^{\infty} x e^{-x} d x=e^{-\frac{1}{\xi}}(2 \xi+1) .
\end{gathered}
$$

Therefore

$$
(*)=1+2\left[e^{-\frac{1}{\xi}}(2 \xi+1)+\left(1+\frac{1}{\xi}\right) e^{-\frac{1}{\xi}}\right]=1+2 e^{-\frac{1}{\xi}}\left(2 \xi+2+\frac{1}{\xi}\right) .
$$

Consequently we have found that

$$
\begin{gathered}
\sum_{v=-\infty}^{\infty}\left(1+\frac{|v|}{\xi}\right) e^{-\frac{|v|}{\xi}}=1+2 \sum_{\nu=1}^{\infty}\left(1+\frac{v}{\xi}\right) e^{-\frac{v}{\xi}} \\
\leq 1+2 e^{-\frac{1}{\xi}}\left(2 \xi+2+\frac{1}{\xi}\right) \text { (finite) } \rightarrow 1 \text {, as } \xi \rightarrow 0+.
\end{gathered}
$$


Finally we observe

$$
\left(P_{\xi}^{*} f\right)(x)-f(x)=\frac{\sum_{v=-\infty}^{\infty}(f(x+v)-f(x)) e^{-\frac{|v|}{\xi}}}{\sum_{v=-\infty}^{\infty} e^{-\frac{|v|}{\xi}}} .
$$

So that

$$
\begin{gathered}
\left|\left(P_{\xi}^{*} f\right)(x)-f(x)\right| \leq \frac{\sum_{v=-\infty}^{\infty}|f(x+v)-f(x)| e^{-\frac{|v|}{\xi}}}{\sum_{v=-\infty}^{\infty} e^{-\frac{|v|}{\xi}}} \\
\leq \frac{\sum_{v=-\infty}^{\infty} \omega_{1}(f,|v|) e^{-\frac{|v|}{\xi}}}{\sum_{v=-\infty}^{\infty} e^{-\frac{|v|}{\xi}}}=\frac{\sum_{v=-\infty}^{\infty} \omega_{1}\left(f, \xi \frac{|v|}{\xi}\right) e^{-\frac{|v|}{\xi}}}{\sum_{v=-\infty}^{\infty} e^{-\frac{|v|}{\xi}}}
\end{gathered}
$$

(by (21))

$$
\leq \omega_{1}(f, \xi)\left(\frac{\sum_{v=-\infty}^{\infty}\left(1+\frac{|v|}{\xi}\right) e^{-\frac{|v|}{\xi}}}{\sum_{v=-\infty}^{\infty} e^{-\frac{|v|}{\xi}}}\right)
$$

(by (14), (19))

$$
\leq \omega_{1}(f, \xi) \frac{\left(1+2 e^{-\frac{1}{\xi}}\left(2 \xi+2+\frac{1}{\xi}\right)\right)}{\left(2 \xi e^{-\frac{1}{\xi}}+1\right)} .
$$

We notice that

$$
\frac{\left(1+2 e^{-\frac{1}{\xi}}\left(2 \xi+2+\frac{1}{\xi}\right)\right)}{\left(2 \xi e^{-\frac{1}{\xi}}+1\right)} \rightarrow 1, \text { as } \xi \rightarrow 0+.
$$

We have proved

$$
\left|\left(P_{\xi}^{*} f\right)(x)-f(x)\right| \leq\left[\frac{1+2 e^{-\frac{1}{\xi}}\left(2 \xi+2+\frac{1}{\xi}\right)}{1+2 \xi e^{-\frac{1}{\xi}}}\right] \omega_{1}(f, \xi),
$$

$\forall x \in \mathbb{R}$

The proof now is completed.

Next we prove preservation of global smoothness of $\mathrm{P}_{\xi}^{*}$.

Theorem 2.2. It holds

$$
\omega_{1}\left(P_{\xi}^{*} f, \delta\right) \leq \omega_{1}(f, \delta), \quad \forall \delta>0 .
$$

Inequality (25) is sharp, namely it is attained by $\mathrm{f}(\mathrm{x})=$ identity $(\mathrm{x})=\mathrm{x}$.

Proof. We see that

$$
\left(P_{\xi}^{*} f\right)(x)-\left(P_{\xi}^{*} f\right)(y)=\frac{\sum_{v=-\infty}^{\infty}(f(x+v)-f(y+v)) e^{-\frac{|v|}{\xi}}}{\sum_{v=-\infty}^{\infty} e^{-\frac{|v|}{\xi}}} .
$$


Hence

$$
\begin{gathered}
\left|\left(P_{\xi}^{*} f\right)(x)-\left(P_{\xi}^{*} f\right)(y)\right| \leq \frac{\sum_{v=-\infty}^{\infty}|f(x+v)-f(y+v)| e^{-\frac{|v|}{\xi}}}{\sum_{v=-\infty}^{\infty} e^{-\frac{|v|}{\xi}}} \\
\leq \frac{\sum_{v=-\infty}^{\infty} \omega_{1}(f,|x-y|) e^{-\frac{|v|}{\xi}}}{\sum_{v=-\infty}^{\infty} e^{-\frac{|v|}{\xi}}}=\omega_{1}(f,|x-y|) .
\end{gathered}
$$

So that for any $x, y \in \mathbb{R}:|x-y|<\delta$ we get (25).

If $f=i d$, then trivially we get

$$
\left(P_{\xi}^{*} i d\right)(x)-\left(P_{\xi}^{*} i d\right)(y)=x-y=i d(x)-i d(y),
$$

thus (25) is attained.

Next we study the approximation properties of discrete Gauss-Weierstrass operators.

Theorem 2.3. Let $f \in \mathrm{C}_{\mathrm{u}}(\mathbb{R}), 0<\xi \leq 1$. Then

$$
\left\|W_{\xi}^{*} f-f\right\|_{\infty} \leq C(\xi) \omega_{1}(f, \sqrt{\xi})
$$

where

$$
C(\xi):=\left[1+\left(\frac{e^{-\frac{1}{\xi}}\left(\sqrt{\xi}+2+\frac{2}{\sqrt{\xi}}\right)}{\sqrt{\pi \xi}\left(1-\operatorname{erf}\left(\frac{1}{\sqrt{\xi}}\right)\right)+1}\right)\right] .
$$

We have $\lim _{\xi \rightarrow 0+} \mathrm{C}(\xi)=1$, and by $\lim _{\xi \rightarrow 0+} \omega_{1}(\mathrm{f}, \sqrt{\xi})=0$, we get $\mathrm{W}_{\xi}^{*} \rightarrow$ I uniformly with rates, as $\xi \rightarrow 0+$.

Proof. We notice easily that

$$
\sum_{\nu=-1}^{-\infty} e^{-\frac{v^{2}}{\xi}}=\sum_{\nu=1}^{\infty} e^{-\frac{v^{2}}{\xi}}<\sum_{\nu=1}^{\infty} \frac{1}{v^{2}}=\frac{\pi^{2}}{6}<\infty
$$

So we can write

$$
\sum_{v=-\infty}^{\infty} e^{-\frac{v^{2}}{\xi}}=2 \sum_{\nu=1}^{\infty} e^{-\frac{v^{2}}{\xi}}+1<\frac{\pi^{2}}{3}+1
$$

Since $e^{-\frac{v^{2}}{\xi}}$ is positive, continuous and decreasing, by [4, we get

$$
\sum_{v=1}^{\infty} e^{-\frac{v^{2}}{\xi}}-e^{-\frac{1}{\varepsilon}} \leq \int_{1}^{\infty} e^{-\frac{v^{2}}{\xi}} d v \leq \sum_{v=1}^{\infty} e^{-\frac{v^{2}}{\xi}} .
$$

So that

$$
2 \int_{1}^{\infty} e^{-\frac{v^{2}}{\xi}} d v+1 \leq 2 \sum_{v=1}^{\infty} e^{-\frac{v^{2}}{\xi}}+1=\sum_{v=-\infty}^{\infty} e^{-\frac{v^{2}}{\xi}}
$$


and

$$
0<\frac{1}{\sum_{v=-\infty}^{\infty} e^{-\frac{v^{2}}{\xi}}} \leq \frac{1}{2 \int_{1}^{\infty} e^{-\frac{v^{2}}{\xi}} \mathrm{d} v+1}
$$

We know that $\int_{0}^{\infty} e^{-t^{2}} d t=\frac{\sqrt{\pi}}{2}$, and $\operatorname{erf}(x):=\frac{2}{\sqrt{\pi}} \int_{0}^{x} e^{-t^{2}} d t$, with erf $(\infty)=1$.

Hence

$$
\begin{gathered}
2 \int_{1}^{\infty} e^{-\frac{v^{2}}{\xi}} \mathrm{d} v+1=2 \sqrt{\xi} \int_{1}^{\infty} e^{-\left(\frac{v}{\sqrt{\xi}}\right)^{2}} \mathrm{~d}\left(\frac{v}{\sqrt{\xi}}\right)+1= \\
2 \sqrt{\xi} \int_{\frac{1}{\sqrt{\xi}}}^{\infty} e^{-\theta^{2}} \mathrm{~d} \theta+1=2 \sqrt{\xi}\left[\int_{0}^{\infty} e^{-\theta^{2}} \mathrm{~d} \theta-\int_{0}^{\frac{1}{\sqrt{\xi}}} e^{-\theta^{2}} \mathrm{~d} \theta\right]+1 \\
=2 \sqrt{\xi}\left[\frac{\sqrt{\pi}}{2}-\frac{\sqrt{\pi}}{2} \operatorname{erf}\left(\frac{1}{\sqrt{\xi}}\right)\right]+1=\sqrt{\pi \xi}\left(1-\operatorname{erf}\left(\frac{1}{\sqrt{\xi}}\right)\right)+1 .
\end{gathered}
$$

Therefore

$$
2 \int_{1}^{\infty} e^{-\frac{v^{2}}{\xi}} \mathrm{d} v+1=\sqrt{\pi \xi}\left(1-\operatorname{erf}\left(\frac{1}{\sqrt{\xi}}\right)\right)+1 \rightarrow 1, \text { as } \xi \rightarrow 0+.
$$

So we got that

$$
0<\frac{1}{\sum_{v=-\infty}^{\infty} e^{-\frac{v^{2}}{\xi}}} \leq \frac{1}{\sqrt{\pi \xi}\left(1-\operatorname{erf}\left(\frac{1}{\sqrt{\xi}}\right)\right)+1} \rightarrow 1, \text { as } \xi \rightarrow 0+
$$

Next we prove that $g(v)=v e^{-\frac{v^{2}}{\xi}}$ is decreasing for $v \geq 1$. Indeed we have $g^{\prime}(v)=e^{-\frac{v^{2}}{\xi}}\left(1-\frac{2 v^{2}}{\xi}\right) \leq$ 0 , iff $1-\frac{2 v^{2}}{\xi} \leq 0$, iff $\xi \leq 2 v^{2}$, which is true.

So that we have (by [4])

$$
\begin{gathered}
\sum_{v=1}^{\infty}\left(1+\frac{v}{\sqrt{\xi}}\right) e^{-\frac{v^{2}}{\xi}} \leq \int_{1}^{\infty}\left(1+\frac{v}{\sqrt{\xi}}\right) e^{-\frac{v^{2}}{\xi}} \mathrm{d} v+\left(1+\frac{1}{\sqrt{\xi}}\right) e^{-\frac{1}{\xi}}= \\
\int_{1}^{\infty} e^{-\frac{v^{2}}{\xi}} \mathrm{d} v+\int_{1}^{\infty} \frac{v}{\sqrt{\xi}} e^{-\frac{v^{2}}{\xi}} \mathrm{d} v+e^{-\frac{1}{\xi}}+\frac{e^{-\frac{1}{\xi}}}{\sqrt{\xi}}= \\
\frac{\sqrt{\pi \xi}}{2}\left(1-\operatorname{erf}\left(\frac{1}{\sqrt{\xi}}\right)\right)+\frac{\sqrt{\xi}}{2} \int_{1}^{\infty} e^{-\frac{v^{2}}{\xi}} \mathrm{d}\left(\frac{v^{2}}{\xi}\right)+e^{-\frac{1}{\xi}}+\frac{e^{-\frac{1}{\xi}}}{\sqrt{\xi}}= \\
\frac{\sqrt{\pi \xi}}{2}\left(1-\operatorname{erf}\left(\frac{1}{\sqrt{\xi}}\right)\right)+\frac{\sqrt{\xi}}{2} \int_{\frac{1}{\xi}}^{\infty} e^{-x} \mathrm{~d} x+e^{-\frac{1}{\xi}}+\frac{e^{-\frac{1}{\xi}}}{\sqrt{\xi}}= \\
\frac{\sqrt{\pi \xi}}{2}\left(1-\operatorname{erf}\left(\frac{1}{\sqrt{\xi}}\right)\right)+\frac{\sqrt{\xi}}{2} e^{-\frac{1}{\xi}}+e^{-\frac{1}{\xi}}+\frac{e^{-\frac{1}{\xi}}}{\sqrt{\xi}} .
\end{gathered}
$$

That is

$$
\sum_{\nu=1}^{\infty}\left(1+\frac{v}{\sqrt{\xi}}\right) e^{-\frac{v^{2}}{\xi}} \leq \frac{\sqrt{\pi \xi}}{2}\left(1-\operatorname{erf}\left(\frac{1}{\sqrt{\xi}}\right)\right)+e^{-\frac{1}{\xi}}\left(\frac{\sqrt{\xi}}{2}+1+\frac{1}{\sqrt{\xi}}\right)
$$

(finite) $\rightarrow 0$, as $\xi \rightarrow 0+$. 
Since

$$
\sum_{v=-\infty}^{\infty}\left(1+\frac{|v|}{\sqrt{\xi}}\right) e^{-\frac{v^{2}}{\xi}}=2 \sum_{v=1}^{\infty}\left(1+\frac{v}{\sqrt{\xi}}\right) e^{-\frac{v^{2}}{\xi}}+1<\infty,
$$

we find

$$
\sum_{v=-\infty}^{\infty}\left(1+\frac{|v|}{\sqrt{\xi}}\right) e^{-\frac{v^{2}}{\xi}} \leq \sqrt{\pi \xi}\left(1-\operatorname{erf}\left(\frac{1}{\sqrt{\xi}}\right)\right)+e^{-\frac{1}{\xi}}\left(\sqrt{\xi}+2+\frac{2}{\sqrt{\xi}}\right)+1
$$

(is finite) $\rightarrow 1$, as $\xi \rightarrow 0+$.

Next we observe that

$$
\left(W_{\xi}^{*} f\right)(x)-f(x)=\frac{\sum_{v=-\infty}^{\infty}(f(x+v)-f(x)) e^{-\frac{v^{2}}{\xi}}}{\sum_{v=-\infty}^{\infty} e^{-\frac{v^{2}}{\xi}}} .
$$

Thus

$$
\begin{gathered}
\left|\left(W_{\xi}^{*} f\right)(x)-f(x)\right| \leq \frac{\sum_{v=-\infty}^{\infty}|f(x+v)-f(x)| e^{-\frac{v^{2}}{\xi}}}{\sum_{v=-\infty}^{\infty} e^{-\frac{v^{2}}{\xi}}} \leq \\
\frac{\sum_{v=-\infty}^{\infty} \omega_{1}(f,|v|) e^{-\frac{v^{2}}{\xi}}}{\sum_{v=-\infty}^{\infty} e^{-\frac{v^{2}}{\xi}}}=\frac{\sum_{v=-\infty}^{\infty} \omega_{1}\left(f, \sqrt{\xi} \frac{|v|}{\sqrt{\xi}}\right) e^{-\frac{v^{2}}{\xi}}}{\sum_{v=-\infty}^{\infty} e^{-\frac{v^{2}}{\xi}}} \leq \\
\frac{\omega_{1}(f, \sqrt{\xi}) \sum_{v=-\infty}^{\infty}\left(1+\frac{|v|}{\sqrt{\xi}}\right) e^{-\frac{v^{2}}{\xi}}}{\sum_{v=-\infty}^{\infty} e^{-\frac{v^{2}}{\xi}}}
\end{gathered}
$$

(by (39), (44))

$$
\begin{gathered}
\leq \omega_{1}(f, \sqrt{\xi})\left(\frac{\sqrt{\pi \xi}\left(1-\operatorname{erf}\left(\frac{1}{\sqrt{\xi}}\right)\right)+e^{-\frac{1}{\xi}}\left(\sqrt{\xi}+2+\frac{2}{\sqrt{\xi}}\right)+1}{\sqrt{\pi \xi}\left(1-\operatorname{erf}\left(\frac{1}{\sqrt{\xi}}\right)\right)+1}\right)= \\
\omega_{1}(f, \sqrt{\xi})\left(1+\frac{e^{-\frac{1}{\xi}}\left(\sqrt{\xi}+2+\frac{2}{\sqrt{\xi}}\right)}{\sqrt{\pi \xi}\left(1-\operatorname{erf}\left(\frac{1}{\sqrt{\xi}}\right)\right)+1}\right) .
\end{gathered}
$$

So we have proved that

$$
\left|\left(W_{\xi}^{*} f\right)(x)-f(x)\right| \leq \omega_{1}(f, \sqrt{\xi})\left(1+\frac{e^{-\frac{1}{\xi}}\left(\sqrt{\xi}+2+\frac{2}{\sqrt{\xi}}\right)}{\sqrt{\pi \xi}\left(1-\operatorname{erf}\left(\frac{1}{\sqrt{\xi}}\right)\right)+1}\right),
$$

$\forall x \in \mathbb{R}$, any $0<\xi \leq 1$.

The constant in the last inequality converges to 1 , as $\xi \rightarrow 0+$.

The proof of the theorem is completed.

It follows the global smoothness preservation property of $W_{\xi}^{*}$. 
Theorem 2.4. It holds

$$
\omega_{1}\left(W_{\xi}^{*} f, \delta\right) \leq \omega_{1}(f, \delta), \forall \delta>0 .
$$

Inequality (51) is sharp, attained by $\mathrm{f}(\mathrm{x})=\mathrm{id}(\mathrm{x})=\mathrm{x}$.

Proof. We see that

$$
\left(W_{\xi}^{*} f\right)(x)-\left(W_{\xi}^{*} f\right)(y)=\frac{\sum_{v=-\infty}^{\infty}(f(x+v)-f(y+v)) e^{-\frac{v^{2}}{\xi}}}{\sum_{v=-\infty}^{\infty} e^{-\frac{v^{2}}{\xi}}}, \forall x, y \in \mathbb{R} .
$$

Hence

$$
\begin{aligned}
& \left|\left(W_{\xi}^{*} f\right)(x)-\left(W_{\xi}^{*} f\right)(y)\right| \leq \frac{\sum_{v=-\infty}^{\infty}|f(x+v)-f(y+v)| e^{-\frac{v^{2}}{\xi}}}{\sum_{v=-\infty}^{\infty} e^{-\frac{v^{2}}{\xi}}} \\
& \leq \omega_{1}(f,|x-y|)\left(\frac{\sum_{v=-\infty}^{\infty} e^{-\frac{v^{2}}{\xi}}}{\sum_{v=-\infty}^{\infty} e^{-\frac{v^{2}}{\xi}}}\right)=\omega_{1}(f,|x-y|), \forall x, y \in \mathbb{R},
\end{aligned}
$$

proving (51). Sharpness is obvious.

Next we study the approximation properties of general discrete Poisson-Cauchy operators.

Theorem 2.5. Let $\mathrm{f} \in \mathrm{C}_{\mathrm{u}}(\mathbb{R}), 0<\xi \leq 1$. Then

$$
\left\|M_{\xi}^{*} f-f\right\| \leq D(\xi) \omega_{1}(f, \xi)
$$

where

$$
\mathrm{D}(\xi):=\left[1+4 \xi^{2 \alpha \beta}\left(\frac{\alpha \beta}{2 \alpha \beta-1}\right)+\xi^{2 \alpha \beta-1}\left(\frac{2 \alpha \beta-1}{\alpha \beta-1}\right)\right] .
$$

We have $\lim _{\xi \rightarrow 0+} \mathrm{D}(\xi)=1$, and by $\lim _{\xi \rightarrow 0+} \omega_{1}(\mathrm{f}, \xi)=0$, we get $\mathrm{M}_{\xi}^{*} \rightarrow \mathrm{I}$ uniformly with rates, as $\xi \rightarrow 0+$.

Proof. Here $0<\xi \leq 1, \alpha \in \mathbb{N}, \beta>\frac{1}{\alpha}, x \in \mathbb{R}$. By [5], p. 397, formula 595, we have

$$
\int_{0}^{\infty} \frac{1}{\left(t^{2 \alpha}+\xi^{2 \alpha}\right)^{\beta}} d t=\frac{\Gamma\left(\frac{1}{2 \alpha}\right) \Gamma\left(\beta-\frac{1}{2 \alpha}\right)}{2 \Gamma(\beta) \alpha \xi^{2 \alpha \beta-1}} .
$$

Clearly $\left(v^{2 \alpha}+\xi^{2 \alpha}\right)^{-\beta}$ is decreasing, continuous and positive for $v \in[1, \infty)$. Hence by $[$, we get

$$
\begin{gathered}
0<\sum_{\nu=1}^{\infty}\left(v^{2 \alpha}+\xi^{2 \alpha}\right)^{-\beta} \leq\left(1+\xi^{2 \alpha}\right)^{-\beta}+\int_{1}^{\infty}\left(v^{2 \alpha}+\xi^{2 \alpha}\right)^{-\beta} \mathrm{d} v \leq \\
\left(1+\xi^{2 \alpha}\right)^{-\beta}+\int_{0}^{\infty}\left(v^{2 \alpha}+\xi^{2 \alpha}\right)^{-\beta} \mathrm{d} v=\left(1+\xi^{2 \alpha}\right)^{-\beta} \\
+\frac{\Gamma\left(\frac{1}{2 \alpha}\right) \Gamma\left(\beta-\frac{1}{2 \alpha}\right)}{2 \Gamma(\beta) \alpha \xi^{2 \alpha \beta-1}}<\infty, \quad \forall \xi \in(0,1] .
\end{gathered}
$$


Consequently we find convergence of

$$
\begin{gathered}
0<S_{1}:=\sum_{v=-\infty}^{\infty}\left(v^{2 \alpha}+\xi^{2 \alpha}\right)^{-\beta}=\xi^{-2 \alpha \beta}+2 \sum_{v=1}^{\infty}\left(v^{2 \alpha}+\xi^{2 \alpha}\right)^{-\beta} \leq \\
\xi^{-2 \alpha \beta}+2\left(1+\xi^{2 \alpha}\right)^{-\beta}+\frac{\Gamma\left(\frac{1}{2 \alpha}\right) \Gamma\left(\beta-\frac{1}{2 \alpha}\right)}{\Gamma(\beta) \alpha \xi^{2 \alpha \beta-1}}<\infty, \quad \forall \xi \in(0,1] .
\end{gathered}
$$

Similarly we have

$$
\sum_{v=1}^{\infty}\left(v^{2 \alpha}+\xi^{2 \alpha}\right)^{-\beta} \geq \int_{1}^{\infty}\left(v^{2 \alpha}+\xi^{2 \alpha}\right)^{-\beta} d v
$$

and

$$
\sum_{v=-\infty}^{\infty}\left(v^{2 \alpha}+\xi^{2 \alpha}\right)^{-\beta} \geq \xi^{-2 \alpha \beta}+2 \int_{1}^{\infty}\left(v^{2 \alpha}+\xi^{2 \alpha}\right)^{-\beta} d v
$$

That is

$$
0<\frac{1}{\sum_{\nu=-\infty}^{\infty}\left(v^{2 \alpha}+\xi^{2 \alpha}\right)^{-\beta}} \leq \frac{1}{\xi^{-2 \alpha \beta}+2 \int_{1}^{\infty}\left(v^{2 \alpha}+\xi^{2 \alpha}\right)^{-\beta} \mathrm{d} v}<\xi^{2 \alpha \beta} .
$$

That

$$
0<\frac{1}{S_{1}}<\xi^{2 \alpha \beta} \rightarrow 0, \text { as } \xi \rightarrow 0+
$$

Hence

$$
\lim _{\xi \rightarrow 0+} \frac{1}{S_{1}}=0
$$

Call $g(v):=v\left(v^{2 \alpha}+\xi^{2 \alpha}\right)^{-\beta}, v \in[1, \infty)$. We have that

$$
g^{\prime}(v)=\left(v^{2 \alpha}+\xi^{2 \alpha}\right)^{-\beta}\left[1-\left(\frac{2 \alpha \beta v^{2 \alpha}}{v^{2 \alpha}+\xi^{2 \alpha}}\right)\right] \leq 0
$$

iff $1-\left(\frac{2 \alpha \beta v^{2 \alpha}}{v^{2 \alpha}+\xi^{2 \alpha}}\right) \leq 0$, iff $v^{2 \alpha}+\xi^{2 \alpha} \leq 2 \alpha \beta v^{2 \alpha}$, iff $\xi^{2 \alpha} \leq v^{2 \alpha}(2 \alpha \beta-1)$, which is true because $2 \alpha \beta-1 \geq 1$ and $v^{2 \alpha}(2 \alpha \beta-1) \geq 1 \geq \xi^{2 \alpha}$. That is $\mathrm{g}$ is decreasing, positive and continuous on $[1, \infty)$

Hence $\left(1+\frac{v}{\xi}\right)\left(v^{2 \alpha}+\xi^{2 \alpha}\right)^{-\beta}$ is decreasing, positive and continuous on $[1, \infty)$.

Thus again by [4] we derive

$$
\begin{gathered}
\sum_{v=1}^{\infty}\left(1+\frac{v}{\xi}\right)\left(v^{2 \alpha}+\xi^{2 \alpha}\right)^{-\beta} \leq \\
\left(1+\frac{1}{\xi}\right)\left(1+\xi^{2 \alpha}\right)^{-\beta}+\int_{1}^{\infty}\left(1+\frac{v}{\xi}\right)\left(v^{2 \alpha}+\xi^{2 \alpha}\right)^{-\beta} \mathrm{d} v .
\end{gathered}
$$

We further notice that

$$
\begin{gathered}
\int_{1}^{\infty}\left(1+\frac{v}{\xi}\right)\left(v^{2 \alpha}+\xi^{2 \alpha}\right)^{-\beta} \mathrm{d} v= \\
\int_{1}^{\infty}\left(v^{2 \alpha}+\xi^{2 \alpha}\right)^{-\beta} \mathrm{d} v+\frac{1}{\xi} \int_{1}^{\infty} v\left(v^{2 \alpha}+\xi^{2 \alpha}\right)^{-\beta} \mathrm{d} v<
\end{gathered}
$$




$$
\begin{gathered}
\int_{1}^{\infty} v^{-2 \alpha \beta} \mathrm{d} v+\frac{1}{\xi} \int_{1}^{\infty} v^{-2 \alpha \beta+1} \mathrm{~d} v=\left(\frac{1}{2 \alpha \beta-1}\right)+ \\
\left(\frac{1}{2 \xi(\alpha \beta-1)}\right)<\infty, \forall \xi \in(0,1] .
\end{gathered}
$$

So that

$$
\begin{gathered}
\sum_{\nu=1}^{\infty}\left(1+\frac{\nu}{\xi}\right)\left(v^{2 \alpha}+\xi^{2 \alpha}\right)^{-\beta}< \\
\left(1+\frac{1}{\xi}\right)\left(1+\xi^{2 \alpha}\right)^{-\beta}+\left(\frac{1}{2 \alpha \beta-1}\right)+\left(\frac{1}{2 \xi(\alpha \beta-1)}\right)<\infty, \forall \xi \in(0,1] .
\end{gathered}
$$

Consequently we obtain

$$
\begin{gathered}
0<S_{2}:=\sum_{\nu=-\infty}^{\infty}\left(1+\frac{|v|}{\xi}\right)\left(v^{2 \alpha}+\xi^{2 \alpha}\right)^{-\beta}= \\
\xi^{-2 \alpha \beta}+2 \sum_{\nu=1}^{\infty}\left(1+\frac{v}{\xi}\right)\left(v^{2 \alpha}+\xi^{2 \alpha}\right)^{-\beta}< \\
\xi^{-2 \alpha \beta}+2\left(1+\frac{1}{\xi}\right)\left(1+\xi^{2 \alpha}\right)^{-\beta}+\left(\frac{2}{2 \alpha \beta-1}\right)+\left(\frac{1}{\xi(\alpha \beta-1)}\right)< \\
\frac{1}{\xi^{2 \alpha \beta}}+2\left(1+\frac{1}{\xi}\right)+\left(\frac{2}{2 \alpha \beta-1}\right)+\left(\frac{1}{\xi(\alpha \beta-1)}\right)=: \varphi(\xi) .
\end{gathered}
$$

So that

$$
0<\mathrm{S}_{2}<\varphi(\xi)<\infty, \forall \xi \in(0,1],
$$

and

$$
\begin{gathered}
0<\frac{S_{2}}{S_{1}} \frac{\sqrt[662]{2}}{<} \xi^{2 \alpha \beta} \varphi(\xi)= \\
1+2 \xi^{2 \alpha \beta}\left(1+\frac{1}{2 \alpha \beta-1}\right)+\xi^{2 \alpha \beta-1}\left(2+\frac{1}{\alpha \beta-1}\right)= \\
{\left[1+4 \xi^{2 \alpha \beta}\left(\frac{\alpha \beta}{2 \alpha \beta-1}\right)+\xi^{2 \alpha \beta-1}\left(\frac{2 \alpha \beta-1}{\alpha \beta-1}\right)\right] \rightarrow 1, \text { as } \xi \rightarrow 0+.}
\end{gathered}
$$

Hence

$$
0<\lim _{\xi \rightarrow 0+} \frac{S_{2}}{S_{1}}<1
$$

Finally we have that

$$
M_{\xi}^{*}(f, x)-f(x)=\frac{\sum_{v=-\infty}^{\infty}(f(x+v)-f(x))\left(v^{2 \alpha}+\xi^{2 \alpha}\right)^{-\beta}}{\sum_{v=-\infty}^{\infty}\left(v^{2 \alpha}+\xi^{2 \alpha}\right)^{-\beta}},
$$

and

$$
\left|M_{\xi}^{*}(f, x)-f(x)\right| \leq \frac{\sum_{v=-\infty}^{\infty}|f(x+v)-f(x)|\left(v^{2 \alpha}+\xi^{2 \alpha}\right)^{-\beta}}{\sum_{v=-\infty}^{\infty}\left(v^{2 \alpha}+\xi^{2 \alpha}\right)^{-\beta}} \leq
$$




$$
\begin{gathered}
\frac{\sum_{v=-\infty}^{\infty} \omega_{1}\left(f, \xi \frac{|v|}{\xi}\right)\left(v^{2 \alpha}+\xi^{2 \alpha}\right)^{-\beta}}{\sum_{v=-\infty}^{\infty}\left(v^{2 \alpha}+\xi^{2 \alpha}\right)^{-\beta}} \leq \\
\omega_{1}(f, \xi)\left(\frac{\sum_{v=-\infty}^{\infty}\left(1+\frac{|v|}{\xi}\right)\left(v^{2 \alpha}+\xi^{2 \alpha}\right)^{-\beta}}{\sum_{v=-\infty}^{\infty}\left(v^{2 \alpha}+\xi^{2 \alpha}\right)^{-\beta}}\right)=\left(\frac{S_{2}}{S_{1}}\right) \omega_{1}(f, \xi) \stackrel{\frac{70 \gamma}{\leq}}{\leq} \\
\leq\left[1+4 \xi^{2 \alpha \beta}\left(\frac{\alpha \beta}{2 \alpha \beta-1}\right)+\xi^{2 \alpha \beta-1}\left(\frac{2 \alpha \beta-1}{\alpha \beta-1}\right)\right] \omega_{1}(f, \xi) .
\end{gathered}
$$

We have derived

$$
\left|M_{\xi}^{*}(f, x)-f(x)\right| \leq\left[1+4 \xi^{2 \alpha \beta}\left(\frac{\alpha \beta}{2 \alpha \beta-1}\right)+\xi^{2 \alpha \beta-1}\left(\frac{2 \alpha \beta-1}{\alpha \beta-1}\right)\right] \omega_{1}(f, \xi),
$$

$\forall x \in \mathbb{R}, \forall \xi \in(0,1]$, proving the claim.

It follows the global smoothness preservation property of $M_{\xi}^{*}$.

Theorem 2.6. It holds

$$
\omega_{1}\left(M_{\xi}^{*} f, \delta\right) \leq \omega_{1}(f, \delta), \quad \forall \delta>0 .
$$

Inequality (76) is sharp, attained by $\mathrm{f}(\mathrm{x})=\mathrm{id}(\mathrm{x})=\mathrm{x}$.

Proof. Similar to the proof of Theorem 2.4.

We continue with

\section{Theorem 2.7. It holds}

$$
\left\|\theta_{\xi}^{*} f-f\right\|_{\infty} \leq\left(\frac{2}{3} \pi^{2} K^{2 \beta}\right) \xi^{2 \beta-1} \omega_{1}(f, \xi) \rightarrow 0 \text {, as } \xi \rightarrow 0+.
$$

Proof. Here we use a lot

$$
\sum_{v=1}^{\infty} \frac{1}{v^{2}}=\frac{\pi^{2}}{6} \quad \text { (Euler, 1741) }
$$

We have

$$
\theta_{\xi}^{*}(f, x)-f(x)=\frac{\xi^{2 \beta} \sum_{v \in \mathbb{Z}-\{0\}}(f(x+v)-f(x))\left(\frac{\varphi\left(\frac{v}{\xi}\right)}{v}\right)^{2 \beta}}{1+\xi^{2 \beta} \sum_{v \in \mathbb{Z}-\{0\}}\left(\frac{\varphi\left(\frac{v}{\xi}\right)}{v}\right)^{2 \beta}} .
$$

Hence

$$
\left|\theta_{\xi}^{*}(f, x)-f(x)\right| \leq \frac{\xi^{2 \beta} \sum_{v \in \mathbb{Z}-\{0\}}|f(x+v)-f(x)|\left(\frac{\varphi\left(\frac{v}{\xi}\right)}{v}\right)^{2 \beta}}{1+\xi^{2 \beta} \sum_{v \in \mathbb{Z}-\{0\}}\left(\frac{\varphi\left(\frac{v}{\xi}\right)}{v}\right)^{2 \beta}}
$$




$$
\begin{gathered}
\leq \frac{\xi^{2 \beta} \sum_{v \in \mathbb{Z}-\{0\}} \omega_{1}\left(f, \xi \frac{|v|}{\xi}\right)\left(\frac{\varphi\left(\frac{v}{\xi}\right)}{v}\right)^{2 \beta}}{1+\xi^{2 \beta} \sum_{v \in \mathbb{Z}-\{0\}}\left(\frac{\varphi\left(\frac{v}{\xi}\right)}{v}\right)^{2 \beta}} \\
\leq \frac{\xi^{2 \beta} \omega_{1}(f, \xi) \sum_{v \in \mathbb{Z}-\{0\}}\left(1+\frac{|v|}{\xi}\right)\left(\frac{\varphi\left(\frac{v}{\xi}\right)}{v}\right)^{2 \beta}}{1+\xi^{2 \beta} \sum_{v \in \mathbb{Z}-\{0\}}\left(\frac{\varphi\left(\frac{v}{\xi}\right)}{v}\right)^{2 \beta}} \\
\leq \frac{\xi^{2 \beta} \omega_{1}(f, \xi) K^{2 \beta}\left(\sum_{v \in \mathbb{Z}-\{0\}}\left(1+\frac{|v|}{\xi}\right) \frac{1}{v^{2 \beta}}\right)}{1+\xi^{2 \beta} \sum_{v \in \mathbb{Z}-\{0\}}\left(\frac{\varphi\left(\frac{v}{\xi}\right)}{v}\right)^{2 \beta}}=:(*) .
\end{gathered}
$$

We observe that

$$
\begin{aligned}
0 \leq S_{2} & :=\sum_{v \in \mathbb{Z}-\{0\}}\left(\frac{\varphi\left(\frac{v}{\xi}\right)}{v}\right)^{2 \beta} \leq K^{2 \beta} \sum_{v \in \mathbb{Z}-\{0\}} \frac{1}{v^{2 \beta}} \\
& =2 K^{2 \beta} \sum_{v=1}^{\infty} \frac{1}{v^{2 \beta}}<2 K^{2 \beta} \sum_{v=1}^{\infty} \frac{1}{v^{2}}=\frac{K^{2 \beta} \pi^{2}}{3} .
\end{aligned}
$$

Thus

$$
0 \leq S_{2} \leq \frac{K^{2 \beta} \pi^{2}}{3} .
$$

So that

$$
1 \leq 1+\xi^{2 \beta} S_{2} \leq 1+\frac{\xi^{2 \beta} K^{2 \beta} \pi^{2}}{3}<\infty, \quad \forall \xi>0 .
$$

That is

$$
0<\frac{1}{1+\xi^{2 \beta} S_{2}} \leq 1
$$

with

$$
\lim _{\xi \rightarrow 0+} \frac{1}{1+\xi^{2 \beta} S_{2}}=1 \text {. }
$$

Consequently it holds

$$
\begin{gathered}
(*) \leq 2 \xi^{2 \beta} \omega_{1}(f, \xi) K^{2 \beta}\left(\sum_{v=1}^{\infty}\left(1+\frac{v}{\xi}\right) \frac{1}{v^{2 \beta}}\right) \\
=2 \xi^{2 \beta} \omega_{1}(f, \xi) K^{2 \beta}\left(\sum_{v=1}^{\infty} \frac{1}{v^{2 \beta}}+\frac{1}{\xi} \sum_{v=1}^{\infty} \frac{1}{v^{2 \beta-1}}\right) \\
\leq 2 \xi^{2 \beta} \omega_{1}(f, \xi) K^{2 \beta}\left(\sum_{v=1}^{\infty} \frac{1}{v^{2}}+\frac{1}{\xi} \sum_{v=1}^{\infty} \frac{1}{v^{2}}\right) \\
=\frac{\xi^{2 \beta} \pi^{2}}{3} \omega_{1}(f, \xi) K^{2 \beta}\left(1+\frac{1}{\xi}\right) \leq \frac{2 \xi^{2 \beta-1} \pi^{2}}{3} K^{2 \beta} \omega_{1}(f, \xi) .
\end{gathered}
$$


We have proved that

$$
\left|\theta_{\xi}^{*}(f, x)-f(x)\right| \leq\left(\frac{2}{3} \pi^{2} K^{2 \beta}\right) \xi^{2 \beta-1} \omega_{1}(f, \xi) \rightarrow 0, \text { as } \xi \rightarrow 0+.
$$

The proof is completed.

Example 2.8. In Theorem 2.7 we can take $\varphi$ to be sine, cosine with $\mathrm{K}=1$.

Theorem 2.9. It holds

$$
\omega_{1}\left(\theta_{\xi}^{*} f, \delta\right) \leq \omega_{1}(f, \delta), \quad \forall \delta>0 .
$$

Inequality (89) is attained by $\mathrm{f}=\mathrm{id}$.

We finish by studying $T_{\xi}^{*}$.

Theorem 2.10. It holds

$$
\left\|T_{\xi}^{*} f-f\right\|_{\infty} \leq\left(\frac{2 \pi^{2} A}{3}\right) \xi^{2 \beta-1} \omega_{1}(f, \xi) \rightarrow 0 \text {, as } \xi \rightarrow 0+.
$$

Theorem 2.11. It holds

$$
\omega_{1}\left(T_{\xi}^{*} f, \delta\right) \leq \omega_{1}(f, \delta), \quad \forall \delta>0 .
$$

Inequality (91) is attained by $\mathrm{f}=\mathrm{id}$.

\section{Proof. of Theorem 2.10.}

We have

$$
\mathrm{T}_{\xi}^{*}(f, x)-f(x)=\frac{\sum_{v \in \mathbb{Z}-\{0\}}(f(x+v)-f(x))\left(\frac{\varphi\left(\frac{v}{\xi}\right)}{\left(\frac{v}{\xi}\right)^{4 \beta}}\right)}{1+\sum_{v \in \mathbb{Z}-\{0\}}\left(\frac{\varphi\left(\frac{v}{\xi}\right)}{\left(\frac{v}{\xi}\right)^{4 \beta}}\right)} .
$$

Thus

$$
\begin{gathered}
\left|\mathrm{T}_{\xi}^{*}(f, x)-f(x)\right| \leq \frac{\sum_{v \in \mathbb{Z}-\{0\}}|f(x+v)-f(x)|\left(\frac{\varphi\left(\frac{v}{\xi}\right)}{\left(\frac{v}{\xi}\right)^{4 \beta}}\right)}{1+\sum_{v \in \mathbb{Z}-\{0\}}\left(\frac{\varphi\left(\frac{v}{\xi}\right)}{\left(\frac{v}{\xi}\right)^{4 \beta}}\right)} \\
\leq \frac{\sum_{v \in \mathbb{Z}-\{0\}} \omega_{1}\left(f, \xi \frac{|v|}{\xi}\right)\left(\frac{\varphi\left(\frac{v}{\xi}\right)}{\left(\frac{v}{\xi}\right)^{4 \beta}}\right)}{1+\sum_{v \in \mathbb{Z}-\{0\}}\left(\frac{\varphi\left(\frac{v}{\xi}\right)}{\left(\frac{v}{\xi}\right)^{4 \beta}}\right)} \\
\leq \frac{\omega_{1}(f, \xi) \sum_{v \in \mathbb{Z}-\{0\}}\left(1+\frac{|v|}{\xi}\right)\left(\frac{\varphi\left(\frac{v}{\xi}\right)}{\left(\frac{v}{\xi}\right)^{4 \beta}}\right)}{1+\sum_{v \in \mathbb{Z}-\{0\}}\left(\frac{\varphi\left(\frac{v}{\xi}\right)}{\left(\frac{v}{\xi}\right)^{4 \beta}}\right)}
\end{gathered}
$$




$$
\begin{gathered}
\leq \frac{\omega_{1}(f, \xi) \sum_{v \in \mathbb{Z}-\{0\}}\left(1+\frac{|v|}{\xi}\right) \frac{A}{\left(\frac{v}{\xi}\right)^{2 \beta}}}{1+\sum_{v \in \mathbb{Z}-\{0\}}\left(\frac{\varphi\left(\frac{v}{\xi}\right)}{\left(\frac{v}{\xi}\right)^{4 \beta}}\right)} \\
=\frac{A \omega_{1}(f, \xi) \xi^{2 \beta} \sum_{v \in \mathbb{Z}-\{0\}}\left(1+\frac{|v|}{\xi}\right) \frac{1}{v^{2 \beta}}}{1+\sum_{v \in \mathbb{Z}-\{0\}}\left(\frac{\varphi\left(\frac{v}{\xi}\right)}{\left(\frac{v}{\xi}\right)^{4 \beta}}\right)} \\
=\frac{2 A \omega_{1}(f, \xi) \xi^{2 \beta} \sum_{v=1}^{\infty}\left(1+\frac{v}{\xi}\right) \frac{1}{v^{2 \beta}}}{1+\sum_{v \in \mathbb{Z}-\{0\}}\left(\frac{\varphi\left(\frac{v}{\xi}\right)}{\left(\frac{v}{\xi}\right)^{4 \beta}}\right)} \\
=\frac{2 A \omega_{1}(f, \xi) \xi^{2 \beta}\left[\sum_{v=1}^{\infty} \frac{1}{v^{2 \beta}}+\frac{1}{\xi} \sum_{v=1}^{\infty} \frac{1}{\left.v^{2 \beta-1}\right]}\right.}{1+\sum_{v \in \mathbb{Z}-\{0\}}\left(\frac{\varphi\left(\frac{v}{\xi}\right)}{\left(\frac{v}{\xi}\right)^{4 \beta}}\right)} \\
\leq \frac{\left(\frac{2 \pi^{2}}{3} A\right) \omega_{1}(f, \xi) \xi^{2 \beta-1}}{1+\sum_{1}(f, \xi) \xi^{2 \beta}\left[\frac{\pi^{2}}{6}+\frac{1}{\xi} \frac{\pi^{2}}{6}\right]} \\
\left(1+\sum_{\left.v \in \mathbb{Z}-\{0\}\left(\frac{\varphi\left(\frac{v}{\xi}\right)}{\left(\frac{v}{\xi}\right)^{4 \beta}}\right)\right)} \leq \frac{\varphi\left(\frac{v}{\xi}\right)}{\left(\frac{v}{\xi}\right)^{4 \beta}}\right.
\end{gathered}
$$

We see that

$$
\begin{gathered}
1+\sum_{v \in \mathbb{Z}-\{0\}} \frac{\varphi\left(\frac{v}{\xi}\right)}{\left(\frac{v}{\xi}\right)^{4 \beta}} \leq 1+A \sum_{v \in \mathbb{Z}-\{0\}} \frac{1}{\left(\frac{v}{\xi}\right)^{2 \beta}} \\
=1+A \xi^{2 \beta} \sum_{v \in \mathbb{Z}-\{0\}} \frac{1}{v^{2 \beta}}=1+2 A \xi^{2 \beta} \sum_{\nu=1}^{\infty} \frac{1}{v^{2 \beta}} \\
<1+2 A \xi^{2 \beta}\left(\sum_{v=1}^{\infty} \frac{1}{v^{2}}\right)=1+\frac{A \xi^{2 \beta} \pi^{2}}{3}<\infty, \quad \forall \xi>0 .
\end{gathered}
$$

That is

$$
1 \leq 1+\sum_{v \in \mathbb{Z}-\{0\}} \frac{\varphi\left(\frac{v}{\xi}\right)}{\left(\frac{v}{\xi}\right)^{4 \beta}}<1+\frac{A \pi^{2} \xi^{2 \beta}}{3}<\infty, \quad \forall \xi>0 .
$$

Also $1+\frac{A \pi^{2} \xi^{2 \beta}}{3} \rightarrow 1$, as $\xi \rightarrow 0+$, that is

$$
\lim _{\xi \rightarrow 0+}\left(1+\sum_{v \in \mathbb{Z}-\{0\}} \frac{\varphi\left(\frac{v}{\xi}\right)}{\left(\frac{v}{\xi}\right)^{4 \beta}}\right)=1 .
$$

Furthermore we have

$$
0<\frac{1}{1+\sum_{v \in \mathbb{Z}-\{0\}} \frac{\varphi\left(\frac{v}{\xi}\right)}{\left(\frac{v}{\xi}\right)^{4 \beta}}} \leq 1
$$


Hence

$$
(*) \leq\left(\frac{2 \pi^{2}}{3} A\right) \omega_{1}(f, \xi) \xi^{2 \beta-1} .
$$

We have proved

$$
\left|\mathrm{T}_{\xi}^{*}(\mathrm{f}, \mathrm{x})-\mathrm{f}(\mathrm{x})\right| \leq\left(\frac{2 \pi^{2} \mathrm{~A}}{3}\right) \xi^{2 \beta-1} \omega_{1}(\mathrm{f}, \xi) \rightarrow 0, \text { as } \xi \rightarrow 0+, \forall x \in \mathbb{R},
$$

proving the claim.

Note 2.12. All estimates of this article are also true for $f \in \mathrm{C}_{\mathrm{b}}(\mathbb{R})$, continuous and bounded functions on $\mathbb{R}$. However the convergences fail if $\mathrm{f}$ is not uniformly continuous.

Received: October 2012. Revised: March 2013.

\section{References}

[1] George Anastassiou and Razvan Mezei, Approximation by Singular Integrals, to appear, Cambridge Scientific Publishers, Cambridge, U.K, 2013.

[2] R.A. DeVore and G.G. Lorentz, Constructive Approximation, Springer-Verlag, N.Y., Heildelberg, 1993.

[3] I. Favard, Sur les multiplicateurs d'interpolation, J. Math. Pures Appl., IX, 23(1944), 219-247.

[4] F. Smarandache, A triple inequality with series and improper integrals, arxiv. org/ftp/math/papers/0605/0605027.pdf, 2006.

[5] D. Zwillinger, CRC standard Mathematical Tables and Formulae, 30th edition, Chapman \& Hall/CRC, Boca Raton, 1995. 